\title{
Population based models of cortical drug response: insights from anaesthesia
}

\author{
Brett L. Foster · Ingo Bojak · David T. J. Liley
}

Received: 9 July 2008/Revised: 28 August 2008/ Accepted: 28 August 2008/Published online: 23 September 2008

(C) The Author(s) 2008. This article is published with open access at Springerlink.com

\begin{abstract}
A great explanatory gap lies between the molecular pharmacology of psychoactive agents and the neurophysiological changes they induce, as recorded by neuroimaging modalities. Causally relating the cellular actions of psychoactive compounds to their influence on population activity is experimentally challenging. Recent developments in the dynamical modelling of neural tissue have attempted to span this explanatory gap between microscopic targets and their macroscopic neurophysiological effects via a range of biologically plausible dynamical models of cortical tissue. Such theoretical models allow exploration of neural dynamics, in particular their modification by drug action. The ability to theoretically bridge scales is due to a biologically plausible averaging of cortical tissue properties. In the resulting macroscopic neural field, individual neurons need not be explicitly represented (as in neural networks). The following paper aims to provide a non-technical introduction to the mean field population modelling of drug action and its recent successes in modelling anaesthesia.
\end{abstract}

Keywords Brain dynamics - Neural field theory · Anaesthesia $\cdot$ EEG $\cdot$ Inhibition

B. L. Foster $(\varangle)$ · D. T. J. Liley

Brain Sciences Institute, Swinburne University of Technology, 400 Burwood Rd., Hawthorn, 3122 Melbourne, Australia e-mail: bfoster@swin.edu.au

D. T. J. Liley

e-mail: dliley@swin.edu.au

I. Bojak

Donders Institute for Brain, Cognition and Behaviour, Department of Cognitive Neuroscience (126), Radboud University Nijmegen Medical Centre, Postbus 9101, 6500 HB Nijmegen, The Netherlands

e-mail: I.Bojak@donders.ru.nl

\section{Introduction}

At present a major difficulty in understanding drug action in the human brain is the ability to relate the multiple physical scales of investigation. How do the findings of molecular biology relate to cellular neurophysiology and in turn to neuroimaging and cognitive testing? It is often only via inference and synthesis that investigators can relate complementary findings across research fields in order to construct a theory. To support such a synthesis, theoretical methods are required to integrate empirical findings. In many respects neuroimaging has sought to do this in regards to cognition and functional neuroanatomy (i.e. by quantifying the relationship between behaviour and measures of functional brain activity). At the microscopic scale there is also success in quantifying the relationship between molecular and cellular levels (e.g. by quantifying the relationship between gene expression and electrophysiology (Hanna 2006)). However, integrating findings from the microscopic (single neuron) level to the macroscopic (neural population) level is underdeveloped (Freeman 1992; Breakspear and Stam 2005). One potential approach is the growing area of mean field population or neural mass modelling (Freeman 1975; Lopes da Silva et al. 1975; Nunez 1981; Jirsa and Haken 1996; Robinson et al. 1997; Liley et al. 2002; David and Friston 2003). By spatially averaging the behaviour of neurons which constitute cytoarchitechtonically defined populations $(\approx$ macrocolumn$)$, mean field models approximate the intervening mesoscopic domain of neural activity. In doing so these models can bridge the explanatory gap between microscopic and macroscopic domains.

What follows is an introduction to the dynamical mean field modelling of cortical drug response, using recent findings from anaesthesia as an example. Focus is placed 
on the physiological relevance and utility of this approach with less emphasis on the details of mathematical formulations, which are often quite involved. Those readers interested in the mathematical details are directed to the cited materials below, particularly (Steyn Ross et al. 1999; Bojak and Liley 2005; Molaee-Ardekani et al. 2007). The remainder of the current section will cover the applicability of mean field modelling to anaesthesia and in doing so begin to highlight the general principles of this approach. Section "Mean field modelling of neural activity" will provide more details of mean field models and their biological basis. In Section "Modelling anaesthesia" the neurophysiological effects of anaesthetics will be introduced along with their pharmacology (Targets of anaesthetic action), followed by a review of network models of anaesthetic action in Section "Cellular network models of anaesthetic action" and a more detailed review of mean field models in Section "Mean field models of anaesthetic action". The clinical relevance of mean field models will be covered in Section "Clinical implications of mean field population models" followed by summary remarks in Section "Conclusion".

The modelling of anaesthetics is an expedient test case of population models for several reasons. Firstly, the clinical utility of anaesthetics has made these drugs a popular research domain, with particular focus on the sites and mechanisms of action (Rudolph and Antkowiak 2004). As will be discussed below this focus on the development of cellular descriptions of action greatly assist the fidelity of model parameterization. Secondly, volatile anaesthetic pharmacology shows a predominate affinity for ligandgated gamma-amino-butyric-acid (GABA) neurotransmission (Franks and Lieb 1994; Rudolph and Antkowiak 2004). It is therefore notable that population models incorporate fast neurotransmitter kinetics (ligand-gated ionic channels) and constitute cell populations not by diverse morphology, but by the chief functional properties of inhibition (I) and excitation (E). Making the dominant cortical neurotransmitter systems (GABA (I) and Glutamate (E), Petroff 2002) primary interests in modelling cortical pharmacology. Thirdly, as the potent influence of anaesthetics is mediated by a dominant neurotransmitter system, its resulting effects on neurophysiology and behaviour are manifestly global. For example, neocortical populations and the related cognitive faculties are progressively suppressed by anaesthetic agents (Heinke and Koelsch 2005). This global influence also fits with the spatial scale of mean field models. In summary, anaesthetic pharmacology is fortuitously accommodated in the biological content of mean field models of cerebral cortex.

Clinical benefits also arise from the development of theoretical descriptions of anaesthesia. With the growing utilization of electroencephalogram (EEG) based depth of anaesthesia (DOA) monitors, the development of real-time drug monitoring in the central nervous system (CNS) is taking shape. As a majority of mean field models aim to simulate the EEG, they are particularly germane to DOA monitoring. For example, recently the effects of alprazolam, a benzodiazepine, were sensitively monitored (offline) using signal processing methods derived from a population theory of EEG (Liley et al. 2003). Furthermore, these findings provided a sensitive and mechanistic description of how the benzodiazepine class of sedative anxiolytics paradoxically increase EEG beta $(15-25 \mathrm{~Hz})$ power in moderate to high concentrations (Liley et al. 2003). This effort at theoretically driven analysis reflects biologically motivated methods, which are hoped to provide more sensitive and efficient measures than data driven approaches.

\section{Mean field modelling of neural activity}

In recent decades both theoretical neuroscience and cognitive science have shown a growing interest in the use of dynamical methods as a research tool (Kelso 1995; van Gelder 1998). Such research utilizes the tools and vocabulary of the mathematics of dynamical systems for the modelling and empirical analysis of electrical brain activity (Freeman 1992; Stam 2005). This emerging field of neurodynamics (Freeman 2000) has particularly strong ties with electroencephalography (EEG) (Freeman 1975; Basar 1980; Nunez 1995) and magnetoencephalography (MEG) (Jirsa and Haken 1996; David and Friston 2003), as the time scales of these measurements (milliseconds) are more amenable to a dynamical description than BOLD (blood oxygen level dependent) fMRI (functional magnetic resonance imaging) and PET (positron emission tomography) in addition to being more closely matched to the time scales of cognition and behaviour.

Oscillatory dynamics of the EEG has been a main area of research focus in neurodynamics. In general terms the theoretical methods employed to understand the dynamical genesis of the EEG can be divided into two essentially separate, though complementary, approaches. The first approach models the activity of large networks of individual model neurons imbued with a range of voltage and ligand dependent ionic conductances (Koch and Segev 1998). While this approach enables an arbitrary level of physiological detail to be included they are limited in as much as the EEG is a macroscopic or bulk property of populations of neurons. Biophysically the EEG arises from the ionic current flowing in response to synchronised synaptic activity. A typical EEG electrode is recording the synaptic currents generated by well over 100,000 pyramidal neurons (Nunez 1981) (Fig. 1). Thus it is simply 


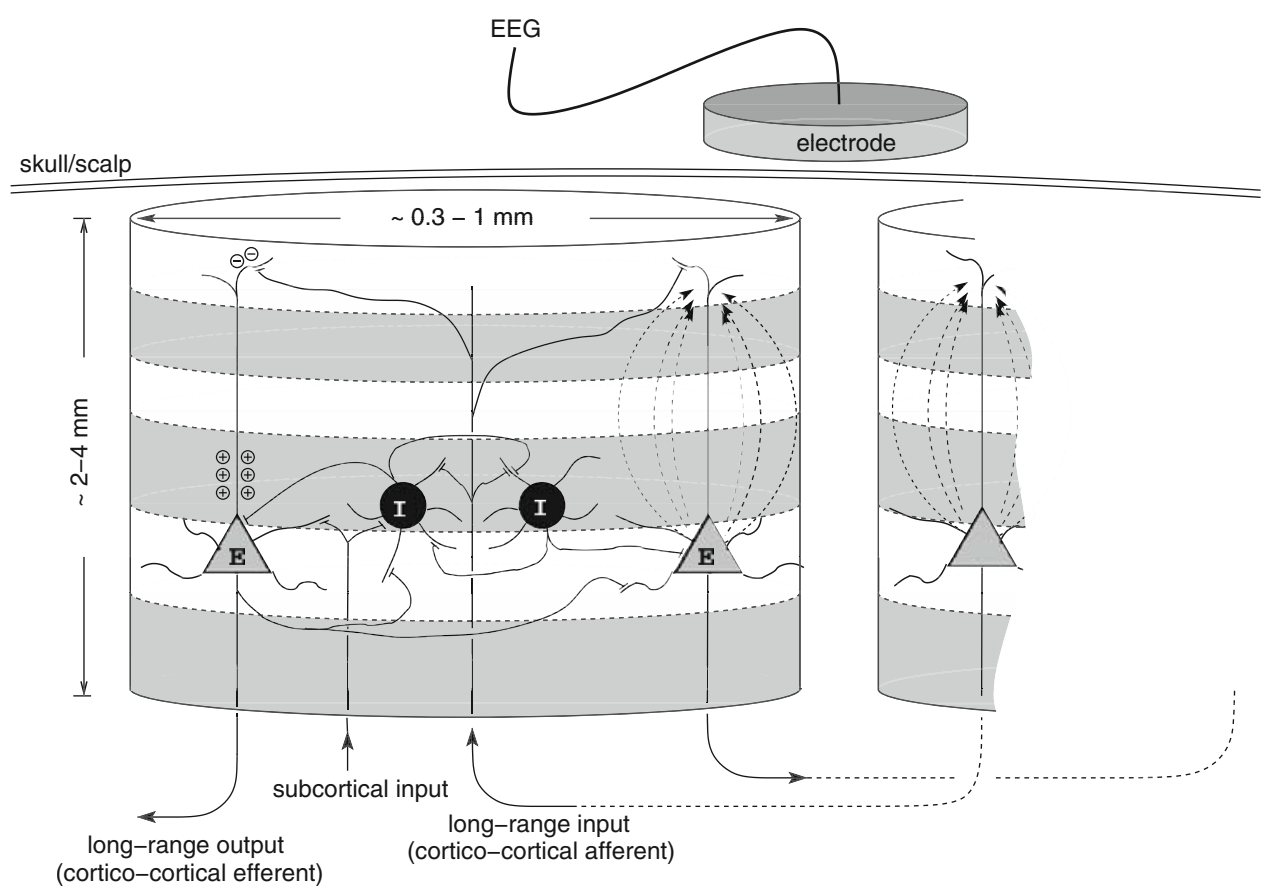

Fig. 1 Schematic representation of a cortical macrocolumn and the two major subtypes of neurons in mammalian cerebral cortexexcitatory (E) and inhibitory (I). Within a macrocolumn excitatory and inhibitory neurons are able to form all possible combinations of feed forward and feedback synaptic connectivity, whereas synaptic connections between cortical macrocolumns are exclusively excitatory. In human neocortex a cortical macrocolumn is estimated to

computationally inefficient to model the EEG using this approach. A preferable approach, in which the spatial scale of the model better reflects the spatial scale of the EEG, is the continuum or mean field model (Nunez 1981; Jirsa and Haken 1996; Robinson et al. 1997; Liley et al. 2002). In this approach it is the synaptic activity generated by spatially circumscribed populations of cortical neurons that are modelled rather than the synaptic activity of individual neurons. In general the columnar organization of cortex (e.g. the macrocolumn) is used to define this spatial scale and to subsequently define the spatial scales of neuronal interactions.

Typical of these mean field theories of electrocortical activity is that of Liley et al. (2002). This theory of EEG is capable of producing the main features of spontaneous human EEG and is able to account quantitatively and qualitatively for the effects that GABA enhancing anaesthetics and sedatives have on the EEG. It assumes that all the various types of cortical neurons can be divided into synaptically interacting excitatory or inhibitory subpopulations. Each of these populations has the spatial extent of a barrel-shaped region of approximately $0.5-3 \mathrm{~mm}$ in diameter penetrating the entire thickness of cortex. Locally (i.e. within a macrocolumn), excitatory and inhibitory contain of the order of 100,000-300,000 neurons (Nunez 1981). Electric field lines, drawn directed along the axis of the apical dendritic trees of the two excitatory neurons on the right, are the result of inward current flow due to excitatory synaptic activity in the distal portions of the apical dendritic tree, and make the greatest contribution to the surface recordable electrocorticogram (ECoG) and EEG. Diagram not draw to scale. Figure from Liley and Bojak (2005)

neuronal populations interact with each other and themselves. Only the excitatory populations are capable of forming connections (long range or cortico-cortical) with excitatory and inhibitory populations within other nearby macrocolumns. This theory, like most other mean field theories, considers the EEG as being proportional to variations of the mean excitatory soma membrane potential $\mathbf{h}_{e}$. The soma membrane is driven from its resting potential $h_{e}^{\mathrm{r}}$ by a weighted sum of synaptic inputs $\mathbf{I}_{l e}$ from excitatory $(e)$ and inhibitory $(i)$ populations:

$\tau_{e} \frac{\partial}{\partial t} \mathbf{h}_{e}=h_{\mathrm{e}}^{r}-\mathbf{h}_{e}+\sum_{l=e, i} \psi_{l e}\left(\mathbf{h}_{e}\right) \mathbf{I}_{l e}$,

with a similar equation describing the inhibitory soma membrane potential $\mathbf{h}_{i}$. In the following a bold quantity $\mathbf{f}$ stands for mean fields depending on position on cortex and time. We will provide here only an outline of the mathematical description, the interested reader can consult (Bojak and Liley 2005; Liley and Bojak 2005) for necessary details. This theory incorporates a number of key physiological properties which are parameterised in such a way that the electroencephalographic effect of changes in these properties by disease or pharmacology can be systematically studied and investigated: 


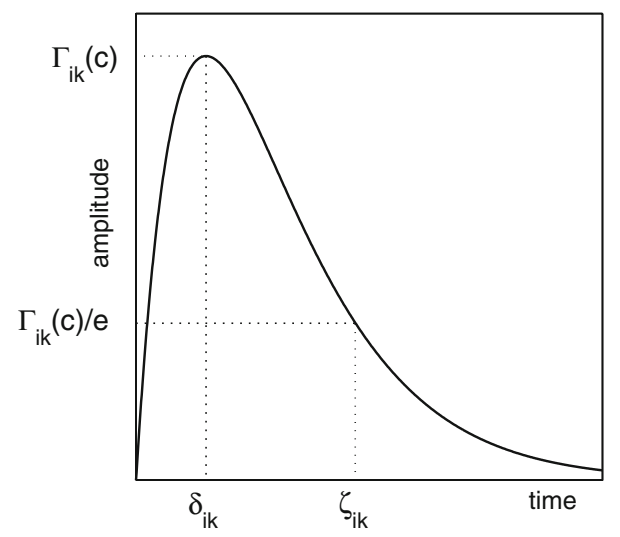

Fig. 2 A schematic illustration of the essential parameters used to characterise the shape and amplitude of an inhibitory-postsynaptic potential (IPSP) or inhibitory postsynaptic current for both theory and experiment. $\Gamma_{i k}$ is the peak amplitude of the IPSP which occurs at time $\delta_{i k}$. The decay constant of the IPSP, $\zeta_{i k}$ is defined as the value at which the IPSP has decayed to approximately $1 / e$ of its peak value. Further details regarding this parameterisation can be found in Liley and Bojak (2005). Figure from Liley and Bojak (2005)

1. Postsynaptic potentials and neurotransmitter kinetics: Neurons are considered as passive electrical compartments into which all synaptically induced ionic current flows. The excitatory and inhibitory synaptic currents, which give rise to excitatory (EPSP) and inhibitory (IPSP) post-synaptic potentials respectively, determine deviations of the mean soma membrane potential from its resting value. In particular the time course and the corresponding reversal potentials of fast ionotropic excitatory (AMPA/kainate) and inhibitory $\left(\mathrm{GABA}_{\mathrm{A}}\right)$ neurotransmitter kinetics are explicitly included. The IPSP response $\mathbf{I}_{i k}$ to presynaptic pulses $\mathbf{P}_{i k}$ is

$\left[\frac{\partial}{\partial t}+\gamma_{i k}\right]\left[\frac{\partial}{\partial t}+\tilde{\gamma}_{i k}\right] \mathbf{I}_{i k}=\Gamma_{i k} \gamma_{i k} e^{\tilde{e}_{i k} \delta_{i k}} \times \mathbf{P}_{i k}$,

and a similar equation applies to the EPSPs $\mathbf{I}_{e k}$. Figure 2 illustrates how the unitary PSP is parameterised and Section "Mean field models of anaesthetic action" below discusses the connection of $\gamma$ and $\tilde{\gamma}$ to the characteristic times $\delta$ and $\zeta$, and their particular significance to the modelling of anaesthesia.

2. Short-range intracortical connectivity: In addition to the reciprocal shortrange excitatory-inhibitory connections intracortical excitatory-excitatory and local inhibitory-inhibitory connections are included-the former because they make up approximately $60 \%$ of all synaptic connections in cortex, the latter because they have been shown to be important determinants of the stability and frequency of alpha band oscillatory activity. Because these connections are short-range (generally less than $3 \mathrm{~mm}$ ) conduction delays are considered negligible. Figure 3 illustrates schematically this topology.

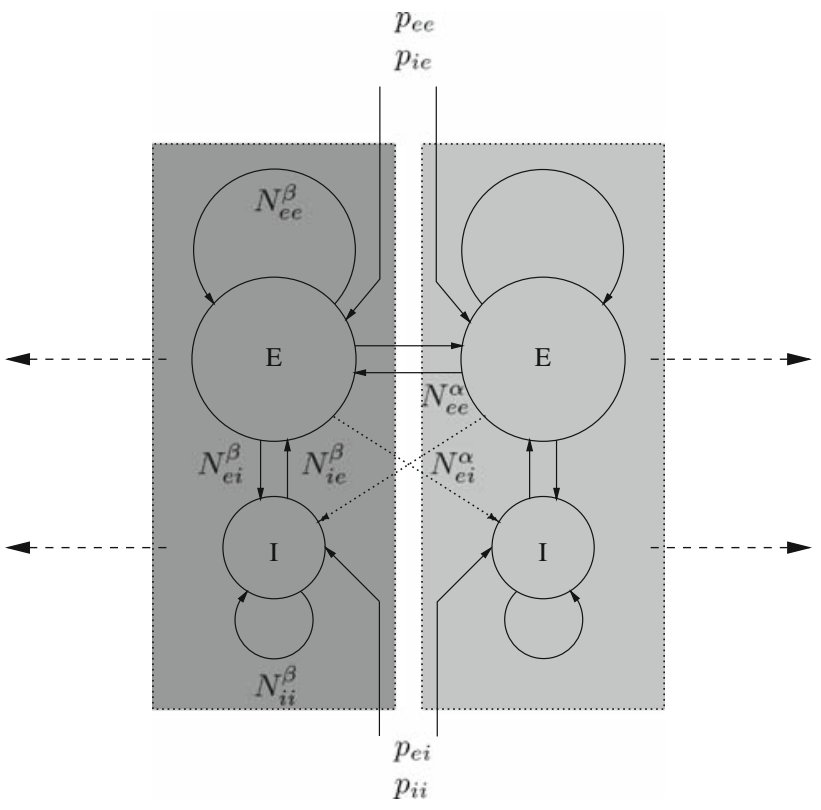

Fig. 3 Schematic illustration of the topology of short-range (intracortical), $N_{j^{\prime} j}^{\beta}$ ), and long-range (cortico-cortical), $N_{e j}^{\alpha}$, fibre systems that serve to connect excitatory (E) and inhibitory (I) neuronal populations in Liley et al.'s dynamical theory of EEG generation (Liley et al. 2002). Populations that are enclosed in a dotted box correspond roughly to a cortical macrocolumn. $p_{j^{\prime} j}$ denotes extracortical input if type $j^{\prime}$ to neural population of type $j$. Figure from Liley and Bojak (2005)

3. Long-range cortico-cortical connectivity: As already mentioned the long range cortico-cortical fibres that serve to connect distant areas of cortex with each other are, on the basis of extensive anatomic and physiological evidence, modelled as being purely excitatory. However because they traverse much larger distances than the intracortical axonal fibres their conduction delays can no longer be considered negligible. The existence of conduction delays is incorporated by ascribing a finite conduction velocity to corticocortical connectivity. The result is a two dimensional telegraph equation

$\left[\left(\frac{1}{v_{e k}} \frac{\partial}{\partial t}+\Lambda_{e k}\right)^{2}-\frac{3}{2} \nabla^{2}\right] \Phi_{e k}=N_{e k}^{\alpha} \Lambda_{e k}^{2} \mathbf{S}_{e}$,

for activity $\Phi_{e k}$ conducted from local excitatory firing $\mathrm{S}_{e}$ to distant excitatory $k=e$ orinhibitory $k=i$ populations.

4. Extra-cortical input: Excitatory or inhibitory thalamic input onto cortical excitatory or inhibitory is incorporated however the theory currently excludes the reciprocal thalamo-cortical connections that a number of other dynamical theories of the EEG include (Robinson et al. 1997) because on the basis of current data their cortical effects cannot be meaningfully parameterised. 
Like the majority of its counterparts (Freeman 1975; Nunez 1981; Jirsa and Haken 1996; Robinson et al. 1997) this theory is cast mathematically as a set of coupled nonlinear partial differential equations. This general mathematical form applies to a wide variety of phenomena in nature, such as the initiation and propagation of the action potential along an axon (the celebrated Hodgkin-Huxley equations) and the flow of compressible and incompressible fluids (the Navier-Stokes equations) (Keener and Sneyd 2001). Because these equations are continuous in space it implies that the macroscopic dynamics of cortex are that of a spatially continuous excitable two dimensional sheet.

This theory, and other continuum mean field theories like it, are capable of producing a range of dynamical activity bearing striking similarity to that actually seen in real EEG and $\mathrm{ECoG}$ recordings. For example the theory of Liley et al. (2002) is able to produce a physiologically plausible alpha rhythm when configured by physiologically admissible parameter sets, as well as producing a rich repertoire of dynamical activity that is expected to accord with the complexity of cognitive activity (Dafilis et al. 2001).

While these mean field theories provide powerful methods for understanding a range of EEG phenomena their real physiological relevance lies in establishing a mesoscopic link between microscopic physiology and macroscopic behaviour (Fig. 4). The advantage of mean field population models of cortical tissue is the ability to explore how perturbations in a range of microscopic parameters influences neural activity macroscopically. For example, exploring how impairing inhibitory neurotransmission microscopically may lead to hyperexcitability at the macroscopic level (e.g. epileptic bursting in EEG) (Kiss and Erdi 2002; Lopes da Silva et al. 2003; Kramer et al. 2007).

\section{Modelling anaesthesia}

Although a variety of phenomena have been explored with population models of the EEG (e.g. epilepsy, sleep, chaos), only recently have researchers highlighted the dynamical modelling of drug effects (Aradi and Erdi 2006). In recent times, a number of researchers have developed detailed mean field descriptions of general anaesthetic drug action (Steyn-Ross et al. 1999; Bojak et al. 2004). These models have not only shown to be capable of simulating unexpected experimental phenomena (e.g. the paradoxical rise and fall in EEG power that occurs during anaesthetic induction-know as the biphasic response (Kuizenga et al. 1998, 2001)), but have also shown the capacity to explain a number of clinical phenomenon (e.g. anaesthetic induced seizure) (Liley and Bojak 2005; Wilson et al. 2006a).

An elementary spectral comparison of the EEG between normal resting states and deep anaesthesia shows a strong suppression of alpha $(8-13 \mathrm{~Hz})$ and beta $(13-25 \mathrm{~Hz})$ power bands, and a dominance of slow wave delta/theta (0.5$8 \mathrm{~Hz}$ ) power (John et al. 2001). Additionally, during deep anaesthesia the EEG signal shows periods of spindle (burst) like oscillations which are interleaved with isoelectric burst suppression (Rampil 1998). These EEG phenomena follow intuitively as a psychophysiological analogue to the well know EEG characterisation of sleep stages (Hobson and Pace Schott 2002). However, the progression from resting state to deep anaesthesia does not appear to be a simple linear depression. A number of researchers have noted that the EEG increases in alpha and/or beta frequencies with low doses of general anaesthetics, particularly propofol (Kishimoto et al. 1995; Feshchenko et al. 2004), high doses of benzodiazepines (Liley et al. 2003; Jensen et al.
Fig. 4 Mean field theories of neural populations are best defined as mesoscopic and thus intervene between microscopic and macroscopic scales. Based on the microscopic study of neurons and their interactions, experimental values are used to parameterise inhibitory and excitatory neurons (a) and their connectivity at intracortical (b), cortico-cortical (c) and extracortical (d) scales. Subsequently these mean values of interaction define summed physiological properties which reflect macroscopic state variables

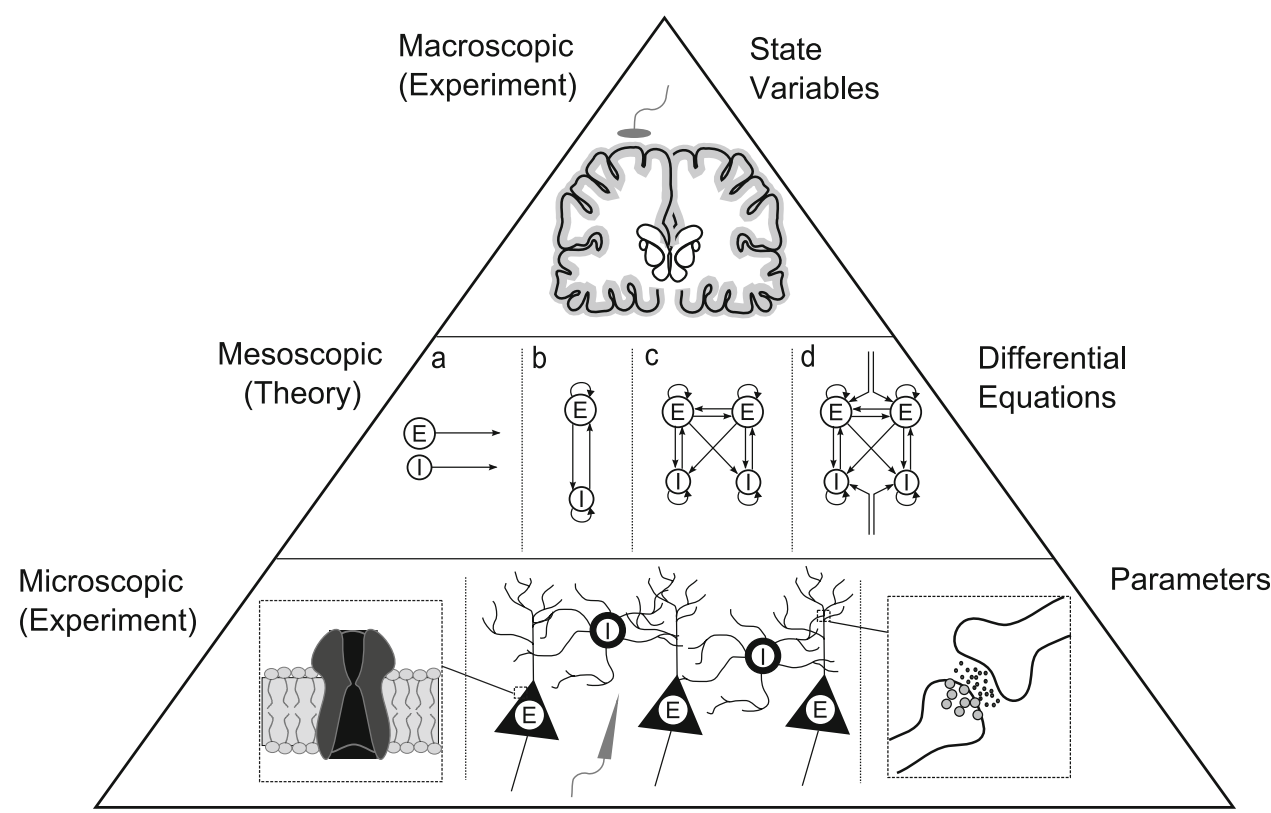


2005) and high doses of dissociative analgesics (Rampil et al. 1998; Tsuda et al. 2007). As mentioned above, a biphasic response has been observed (Kuizenga et al. 1998, 2001), where band limited amplitude increases and decays with anaesthetic induction, and reverses with emergence. Thus, a set of clear quantitative EEG (QEEG) phenomena exist for empirical comparison with theoretical simulation of anaesthesia (John et al. 2001). Furthermore, these empirical phenomenon highlight the dependence of QEEG DOA monitoring upon the capacity to detect the nuance of anaesthetic drug effect, in order to claim sensitivity.

Targets of anaesthetic action

Anaesthesia is generally defined by loss of consciousness, immobility, amnesia and lack of response to noxious stimuli (e.g. incision) (Ishizawa 2007), brought about by a diverse range of neuropharmacological action. However, the predominant pharmacology of general anaesthetics in cerebral cortex is thought to be agonism of $\mathrm{GABA}_{\mathrm{A}}$ ionotropic receptor function (Krasowski and Harrison 1999). Specifically, anaesthetics varyingly potentiate the amplitude and time course of inhibitory post-synaptic potentials (IPSP) via positive allosteric modulation of the $\mathrm{GABA}_{\mathrm{A}}$ receptor (Hemmings et al. 2005). This positive influence, in a simplified sense, imposes a net increase in inhibitory neurotransmission (increased $\mathrm{Cl}^{-}$conductance) and thus a depression of the CNS (Rudolph and Antkowiak 2004). Having a heteropentameric structure the $\mathrm{GABA}_{\mathrm{A}}$ receptor contains a large subunit/subtype diversity (Fig. 5). The predominate stoichiometry is of the form $2 \alpha_{1} 2 \beta_{2} 1 \gamma_{2}$ (Rudolph and Antkowiak 2004). Recent findings in molecular biology have suggested that particular subunit compositions of the $\mathrm{GABA}_{\mathrm{A}}$ receptor mediate general anaesthesia and its sedative/hypnotic influence (Nelson

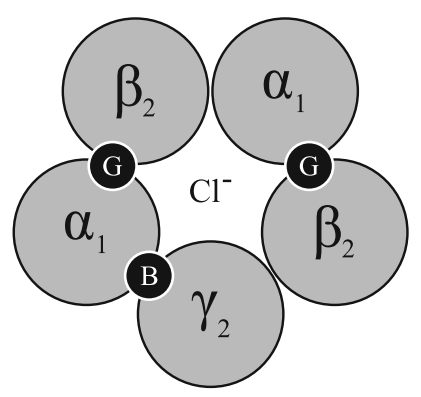

Fig. 5 GABA ionotropic receptors $\left(\mathrm{GABA}_{\mathrm{A}}\right.$ and $\left.\mathrm{GABA}_{\mathrm{C}}\right)$ can be formed via combinations of $\alpha_{(1-6)}, \beta_{(1-4)}, \gamma_{(1-3)}, \delta_{(1)}, \varepsilon_{(1)}, \pi_{(1)}, \theta_{(1)}$ and $\rho_{(1-3)}$ subunits (Sieghart and Sperk 2002). Predominately GABA receptors are of the $2 \alpha_{1} 2 \beta_{2} 1 \gamma_{2}$ isoform, as shown above (McKernan and Whiting 1996; Johnston et al. 1999). Resulting in a $\mathrm{Cl}^{-}$specific ion channel that is gated by GABA, shown above as G. Benzodiazepines modulate $\mathrm{GABA}_{\mathrm{A}}$ receptors via a different binding location, represented as $\mathrm{B}$. Anaesthetics show preference for the $\beta_{2}$ subunit (Campagna-Slater and Weaver 2007) et al. 2002; Reynolds et al. 2003). In particular benzodiazepines may be sensitive to $\gamma$ and $\alpha$ subtypes, whilst anaesthetics differ in affinity relative to $\beta$ subtype (Rudolph and Antkowiak 2004).

In modelling anaesthetic action, investigators need to pay attention to these differences in agent affinity, receptor composition and postsynaptic physiological influence. In the case of current mean field models it is mainly the shape and amplitude of the IPSP which is parametrically altered to model anaesthetic effects (see Fig. 2). Due to the considerable diversity of channel composition and lack of empirical data, individual receptor kinetics cannot be comprehensively modelled at present. In the future more detailed experimental investigations may make this feasible. However, differences between anaesthetic agents and their $\mathrm{GABA}_{\mathrm{A}}$ receptor subtype affinity may well be accounted for sufficiently by their precise impact on the IPSPs alone. As will be highlighted in Section "Clinical implications of mean field population models", minor differences in IPSP shape can lead to significant macroscopic effects.

\section{Cellular network models of anaesthetic action}

Before exploring some of the details of mean field models of anaesthesia, a brief summary of the more discrete cellular and network models will provide some of the principles necessary to parameterise drug action. The chief aim of cellular approaches is to successfully create discrete models of spiking neurons which can be subject to analytical or numerical exploration (Arhem et al. 2003). It is through the coupling of neurons (nodes) that larger cell assemblies/networks can be explored in a similar fashion.

A key example of the cellular/network approach to modelling anaesthesia is the work of Traub et al. (1999) and Faulkner et al. (1998, 1999). Traub et al. (1999) applied their work on hippocampal gamma oscillations to anaesthetics, benzodiazepines and pro-convulsants (thiopentone, diazapam and bicuculline respectively). Simulations of highly coupled inhibitory networks (96 allto-all connected interneurons), simulated empirical findings obtained from hippocampus. Specifically, the authors found that the interneuron network peak field potential frequency (predominantly gamma) was sensitive to the parameter $\tau_{\mathrm{GABA}(\mathrm{A})}$, the decay time constant of hyperpolarising $\mathrm{GABA}_{\mathrm{A}}$ receptor-mediated conductance. Increases in $\tau_{\mathrm{GABA}(\mathrm{A})}$ modelled benzodiazepine and anaesthetic action, consistent with postsynaptic hyperpolarisation and lowered network local field potential frequency. Conversely, the reduction of $\tau_{\mathrm{GABA}(\mathrm{A})}$ reflected those agents which block or otherwise antagonise the $\mathrm{GABA}_{\mathrm{A}}$ receptor (pro-convulsants), leading to hyperexcitability and increased network frequency. One interesting development of these findings relates to capacity of high dose 
benzodiazepines to increase EEG beta power. Traub et al. (1999) speculated that increased beta activity after the ingestion of benzodiazepines may be due to the frequency of background gamma power being lowered, more synchronous and larger in amplitude thus making it more easily detectable at the scalp. These simulation studies may prove to be helpful in understanding similar phenomena induced by low dose intravenous anaesthetics and high dose dissociative anaesthetics (e.g. propofol and ketamine respectively).

In their studies Faulkner et al. explored the influence of anaesthetics upon rat hippocampal slices, both empirically and computationally. In the first of two studies Faulkner et al. explored the influence of anaesthetic and analgesic agents (thiopental, diazepam/temazepam, morphine and ketamine) on elicited synchronous gamma $(40 \mathrm{~Hz})$ oscillations in rat hippocampus (CA1). Complementary computational simulations used a neural network of individual excitatory and inhibitory layers (each being $30 \times 20$ nodes). Nodes were then coupled with 50 excitatory and 49 inhibitory inputs, and a subsection (slice) of the array selected for simulation $(6 \times 20 ; 120$ pyramidal; 120 interneurons). Therefore from a model topology which approximated the CA1 hippocampal morphology, a subsection of this model (slice) could then be compared with the results obtained from experimental hippocampal slice preparations. Baseline oscillatory activity was created though tonic excitatory driving of both cell groups, with inhibitory drug effects modelled by modifying $\mathrm{GABA}_{\mathrm{A}}$ conductance.

Faulkner et al. showed strong similarities between slice recordings and computational simulations of thiopental's suppressive influence on gamma synchrony. The authors postulated that decreased gamma synchrony lowered distributed microscopic communication in the hippocampus, providing a putative amnestic mechanism. Extrapolations of this mechanism therefore suggests that anaesthesia results from wide spread loss of high frequency synchronized network activity. Similar findings were presented by Faulkner et al. (1999), when investigating beta oscillatory synchronisation.

As highlighted above anaesthetic agents are sensitive to changes in the specific subunit composition of the $\mathrm{GABA}_{\mathrm{A}}$ receptor. In a recent investigation Ujfalussy et al. (2007) combined experimental and computational methods to explore $\mathrm{GABA}_{\mathrm{A}}$ receptor $\alpha$ subunit sensitivity of two positive allosteric modulators (zolpidem and L-838417). Computational models were constructed to simulate temporal pacemaker properties of the septo-hippocampal system. Of particular interest were the methods used to model drug effect. For zolpidem, Ujfalussy et al. (2007) assumed that the maximal synaptic conductance of all $\mathrm{GABA}_{\mathrm{A}}$ ionotropic receptor channels in hippocampus
(CA1) and medial septum would increase. Therefore, either the inhibitory time (decay) constant or amplitude of the IPSP was modified to increase the net charge transfer (synaptic current). In contrast, the other agent modelled, L-838417, had specific influence on hippocampal pyramidal neurons and was therefore modelled by lowering the driving phasic input of septo-hippocampal projecting neurons.

Results showed that the simulated effect of zolpidem and L-838417 produced differing patterns of spike and oscillatory behaviour, however both reduced the commonly observed theta oscillation of the septo-hippocampal circuit (Ujfalussy et al. 2007). As zolpidem binds to $\alpha_{1} \mathrm{GABA}_{\mathrm{A}}$ receptors and L-838417 to $\alpha_{2 / 3 / 5} \mathrm{GABA}_{\mathrm{A}}$ receptors, these methods can assist in drug development. In this particular case, the authors suggest that some of the side effects of the broadly binding benzodiazepines (e.g. diazepam) may be avoided by compounds with more specific anxiolytic GABAergic targets (Ujfalussy et al. 2007). This study highlighted the capacity to not only simulate drug action by specific pharmacological parameterisation, but also through altering specific cell group connectivity (i.e. the tonic driving strength of excitatory pyramidal neurons).

\section{Mean field models of anaesthetic action}

Over the past 5-10 years a small number of research groups have focused on developing mean field population models of anaesthetic action, which Sceniak and MacIver (2006) referred to as anaesthesia in silico in a recent editorial. These mesoscopic models are complimentary to single neuronal network based methods, as they allow collective properties of neural tissue dynamics to be simulated on much larger scales. Thus it can be said that while discrete approaches to macroscopic cortical modelling are impractical, so too are the application of population field models to the modelling of microscopic circuits.

The first reported exploration of anaesthetic action in a mean field population model was that of Steyn-Ross et al. (1999). In this work Steyn-Ross et al. (1999) used the model of Liley et al. (1998) to explore the dynamical evolution of EEG activity during simulated anaesthesia. Steyn-Ross et al. (1999) simplified the EEG field theory of Liley et al. (1998) to a set of equations which facilitated analytical exploration using a range of established stochastic methods. With this new set of equations Steyn-Ross et al. (1999) aimed to simulate the effects of anaesthesia by systematically modulating parameters that correspond to the known pharmacological targets associated with anaesthetic action. In particular, of the many system parameters Steyn-Ross et al. (1999) specifically modulated the inhibitory neurotransmitter rate constant $\left(\gamma_{i}\right)$, which quantifies the shape of the IPSP. As highlighted above, general anaesthetics typically exert their influence by positive 
allosteric modulation of the $\mathrm{GABA}_{\mathrm{A}}$ receptor, increasing negative charge transfer and prolonging the tail of the unitary IPSP. Thus reducing $\gamma_{i}$, which increases the time scale of inhibitory neurotransmission, models the increasing effects of anaesthetics. Therefore, anaesthesia was parameterised by scaling the inhibitory neurotransmitter rate constant relative to empirical findings of IPSP prolongation by anaesthetics (Franks and Lieb 1994). By observing how the state variables equivalent to the EEG (excitatory mean soma membrane potential; $h_{e}$ ) changed with modelled increases in drug concentration, the effect of anaesthesia on the EEG was simulated.

Steyn-Ross et al. (1999) found that through the modulation of the inhibitory neurotransmitter rate constant, the model showed three stages of analytical equilibrium which they termed: coma (I), wakefulness (II) and epilepsy (III)

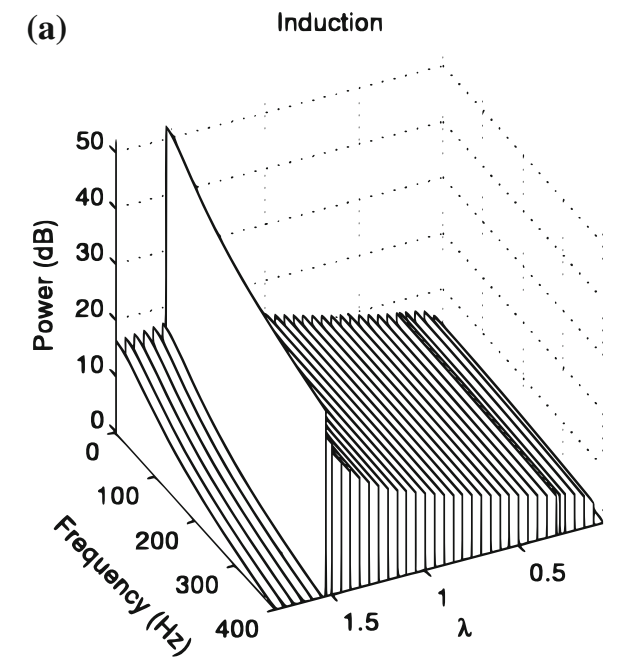

(c)

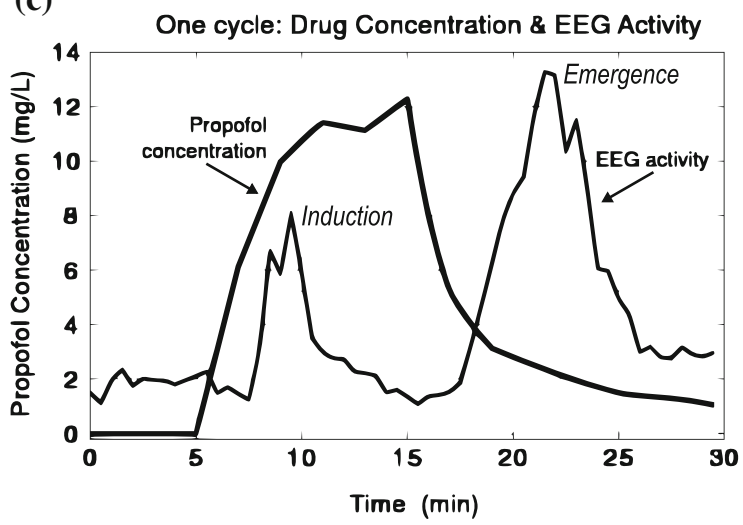

(Fig. 6d). Each of these three system states reflected a progressive reduction of anaesthetic concentration. High levels of modelled anaesthesia (large prolonging of IPSP) produced a state neurophysiologically equivalent to coma or very deep anaesthesia. Subsequently, approximate zero levels of simulated anaesthetic effect produced wakefulness, which in turn could be perturbed to an epileptic like state when anaesthetic influence became negative (equivalent to simulating a pro-convulsant compound which antagonises the $\mathrm{GABA}_{\mathrm{A}}$ IPSP, e.g. bicuculline). SteynRoss et al. (1999) also were able to model a number of important empirical EEG phenomena associated with anaesthetic action, including the biphasic effect (Fig. 6). As indicated above when comparing normal resting levels with deep anaesthesia the EEG shows strong slow wave (delta/theta) dominance. However, the transition between
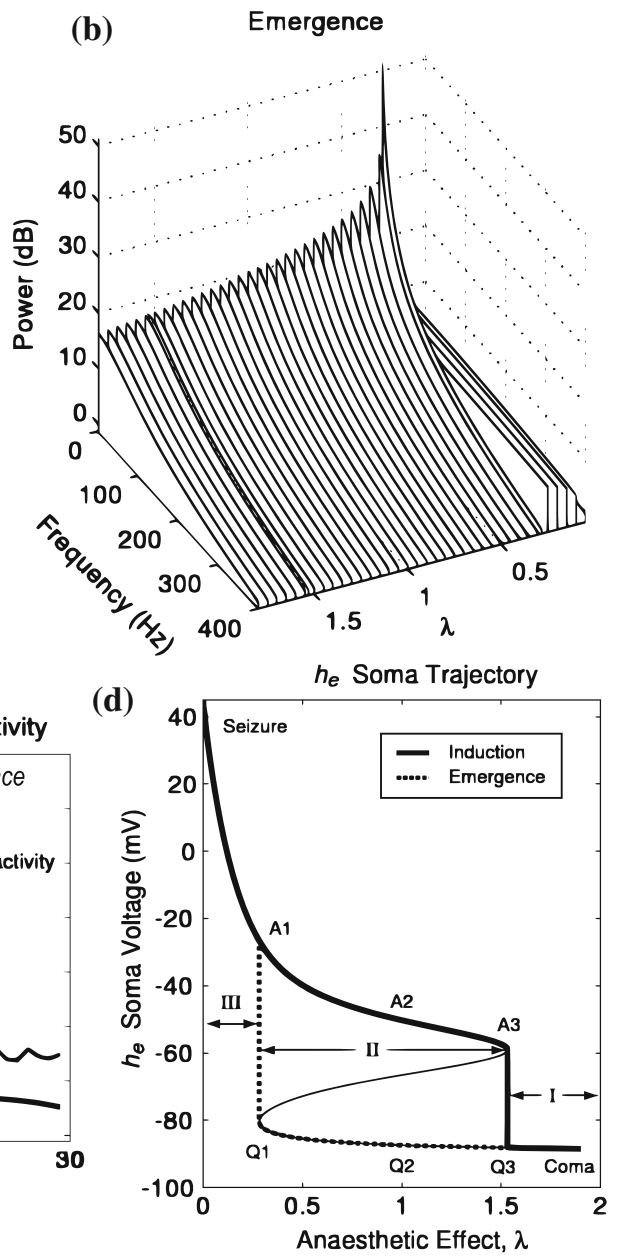

Fig. 6 Steyn-Ross et al. (2004) modelled the abrupt changes in EEG spectral power that have been commonly observed to occur during anaesthetic induction and emergence. (a) the abrupt biphasic response in total modelled EEG spectral power during simulated anaesthetic induction. Anaesthetic action was modelled as a prolongation of the duration of the GABAergic IPSP ( $\lambda$ ). (b) Biphasic response in total EEG spectral power during simulated anaesthetic emergence. (c) Actual EEG recordings of anaesthetic induction and emergence with the intravenous anaesthetic agent propofol which illustrate clearly the biphasic variations in total EEG power as anaesthetic concentration is increased and decreased. (d) Steyn-Ross et al. propose that anaesthetic induced variations in EEG spectral power can be explained theoretically by a stochastically driven system switching between two stable states via a saddle node bifurcation caused by anaesthetic induced variations in IPSP duration. Figure adapted from Figs. 3-5 of Steyn-Ross et al. (2004), with permission 
these two states shows an unexpected rise and fall of band limited ( $\approx 11-15 \mathrm{~Hz}$ ) amplitude, hence the term biphasic response (Kuizenga et al. 1998, 2001). Steyn-Ross et al. (1999) found that modelled EEG spectra also showed a clear biphasic response during simulated anaesthesia.

The primary conclusion of Steyn-Ross et al. (1999) was that the empirical biphasic response could be understood as a parametrically induced phase transition between two dynamically distinct states. Steyn-Ross et al. (1999) went on to further develop their phase transition description of anaesthesia, drawing analogy to the phase transitions commonly seen in a range of thermodynamic systems, such as the sudden transition of a liquid to a gas during heating (Steyn-Ross et al. 2001a, b). Specifically, Steyn-Ross et al. (2001b) developed an excitation parameter which reflected the inverse of anaesthetic effect, and therefore constituted the analogue of heat. It was through a thorough analytical analysis of changes in excitation (heat) that Steyn-Ross et al. (2001b) constructed a dynamical mean field description of anaesthesia. As with thermodynamic phase transitions, Steyn-Ross et al. (2001b) described changes in entropy which coincided with the transition to unconsciousness. The authors justify the physiological relevance of decreases in entropy after the phase transition to unconsciousness (low excitation and strong anaesthetic effect), by pointing to empirical findings of lowered metabolic state (Stullken et al. 1977) and lowered EEG spectral entropy during anaesthesia.

As Steyn-Ross et al. (1999) had used a simplified and spatially lumped version of the Liley et al. (1998) mean field continuum theory, some of the qualitative characteristics of EEG dynamics were not preserved. For example, the canonical alpha rhythm produced by the Liley et al. (1998) model was not maintained, nor was its spatial extent. Therefore in order to begin exploration of the spatial character of the dynamic changes induced by anaesthesia, SteynRoss et al. (2003) constructed a one-dimensional cortex of linked neural masses (cortical rod). This theoretical construction allowed for rudimentary comparison of spatial inhomogeneities during anaesthetic induction (i.e. the theoretical comparison of two separated monopolar electrodes, with a common reference) (Steyn-Ross et al. 2003). Again Steyn-Ross et al. (2003) related the findings of their analytical work to recent discoveries in anaesthesia research, highlighting their theoretical findings of increased spatial covariance as being supported by those empirical results showing increased inter-electrode coherence at the points of loss and recovery of consciousness (i.e. the points of proposed phase transition) (John et al. 2001).

In summary Steyn-Ross et al. (2004) posited that the transition from consciousness to unconsciousness occurs as a consequence of a phase transition in cortical neural population dynamics brought about due to the increasing inhibitory effects of modelled anaesthetic concentration. During this transition there is a surge in band limited EEG power which is of greater magnitude during emergence than during induction, with this hysteresis being observed empirically. Furthermore, the phase transition is also characterised by increased spatial and temporal correlation, for both induction and emergence, with cortical entropy being lowered during unconsciousness. On this basis the authors argue that anaesthetics induce a sharp rise in EEG power that produces a rapid shift into a state of slowed and more ordered EEG rhythmicity, which is reversed, though not symmetrically, with emergence.

While the results of Steyn-Ross et al. were able to account for a number of important macroscopic EEG properties associated with anaesthetic action, they did so in a simplified model that was unable to produce normal resting oscillatory behaviour, such as alpha. In order to remedy this Bojak et al. (2004) used the full set of Liley et al. (2002) equations to model the effects of anaesthetics on the EEG. Unlike the original investigations of SteynRoss et al. (1999), Bojak et al. (2004) did not transform the Liley et al. (2002) equations, rather the full set of equations where numerically solved on a spatio-temporal scale that would more accurately approximate observed EEG. In doing so, the influence of microscopically defined parameter modification could be observed on a macroscopic scale that is comparable to empirical EEG. Subsequently, Bojak et al. (2004) were also able to qualitatively reproduce the biphasic response through only the modulation of the inhibitory decay constant (IPSP prolongation).

A further development in Bojak and Liley (2005) was the reparametrisation of postsynaptic potentials to more accurately fit experimental data. Prior the so-called 'alpha form' had been used, i.e., for an incoming presynaptic pulse at time $t=0$ the assumed PSP response at $t \geq 0$ was:

$R_{\alpha}(t)=\Gamma \gamma \exp (1) \times t \exp (-\gamma t)$,

As illustrated in Fig. 2 for an IPSP, this response has a maximum amplitude $\Gamma$ at rise time $\delta \equiv 1 / \gamma$. Unfortunately the PSP decay time $\zeta$ for which the response falls again to $\Gamma / \exp (1)$ is for the 'alpha form' fixed at $\zeta \simeq 3.1462 \times \delta$. Anaesthetic agents often potentiate $\mathrm{GABA}_{\mathrm{A}}$ induced currents and/or attenuate glutamate mediated ionic currents (Rudolph and Antkowiak 2004), thereby selectively prolonging the inhibitory $\zeta_{i k}$ without affecting the inhibitory rise time $\delta_{i k}$. Hence Bojak and Liley (2005) introduced Eq. 2 yielding a response of the form

$R(t)=\Gamma \gamma \exp (\tilde{\gamma} \delta) \times \frac{\exp (-\gamma t)-\exp (-\tilde{\gamma} t)}{\tilde{\gamma}-\gamma}$,

with $\delta \equiv \ln (\tilde{\gamma} / \gamma) /(\tilde{\gamma}-\gamma)$. This bi-exponential has the same maximum amplitude $R(t=\delta)=\Gamma$. However, the additional parameter $\tilde{\gamma}$ can be used to adjust the decay time 
freely. This allowed Bojak and Liley (2005) to directly incorporate into their model experimental data on the effect of isoflurane on PSPs (MacIver et al. 1996; Banks and Pearce 1999; Nishikawa and MacIver 2000), and to predict the EEG under anaesthesia by calculating the corresponding changes to the excitatory mean soma membrane potential $\mathbf{h}_{e}$, see Eq. 1.

Bojak and Liley (2005) were able to show systematic changes in modelled EEG as a function of isoflurane concentration. An additional selection criteria was then imposed upon these sets of data, which aimed at extracting sets producing a clear biphasic response (Bojak and Liley 2005). The outcomes of Bojak and Liley's (2005) explorations showed some difference to that of Steyn-Ross et al. (2004). It is particularly worth highlighting that while both theories describe changes in macroscopic dynamics during anaesthesia, Steyn-Ross et al. (2004) suggest a phase transition (e.g. boiling water to steam), whereas Bojak and Liley (2005) posit a continuum transition. Bojak and Liley (2005) highlight that the sharp rise in neural firing rate required for Steyn-Ross et al.'s (2004) phase transition is not supported by empirical findings of smooth suppression of action potential frequency with progressive anaesthesia.

Most recently Molaee-Ardekani et al. (2007) sought to further develop the work of Steyn-Ross et al. (2004) and Bojak and Liley (2005), by implementing a model parameterisation of slow voltage dependent mechanisms on excitatory firing rate dynamics. The common sigmoidal relationship between mean soma membrane potential and mean firing rate was modified to included the influence of slow shifts in membrane potential. This voltage dependant slow ionic leak, was motivated by the empirically observed switching (i.e. up and down states) in firing rates of neurons during anaesthesia and non-REM sleep, thought to be controlled by cation conductance. Molaee-Ardekani et al. (2007) explored progressive increases in simulated desflurane anaesthesia, showing up state dominance during waking levels, up/down state switching (delta) at moderate anaesthesia and down state predominance during strong anaesthetic effect (Fig. 7). This switching in firing rate between bursting up states and quiescent down states relates biophysically to the synchronised delta power local field potential predominately seen during deep anaesthesia and stage $3 / 4$ sleep. These findings further promote the ability to theoretically relate cellular experimental observations with bulk macroscopic cortical dynamics via mean field theoretics.

\section{Clinical implications of mean field population models}

The volatile general anaesthetic enflurane, among others, has been shown to paradoxically promote epileptiform

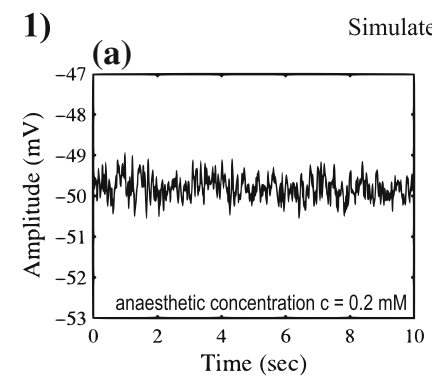

Simulated EEG
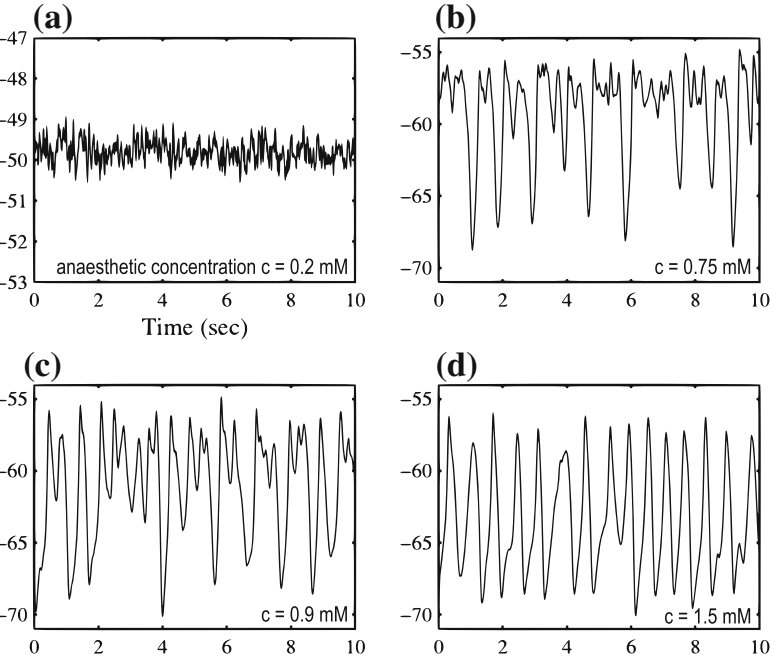

(d)

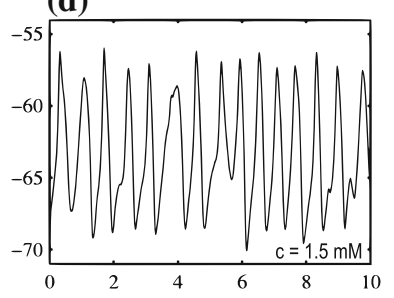

2)

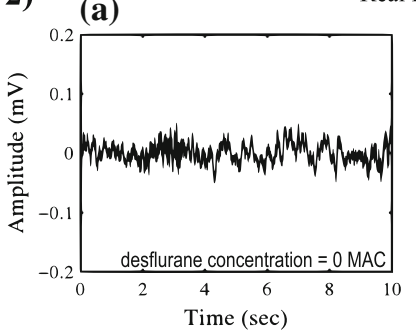

(b)

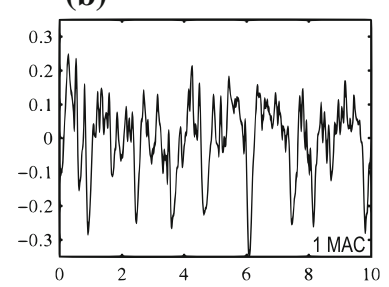

(c)

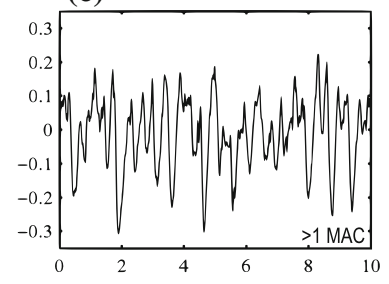

(d)

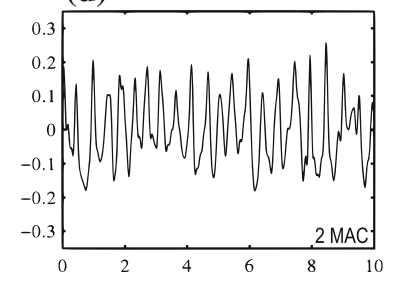

Fig. 7 (1) Molaee-Ardekani et al. (2007) simulated EEG (mean soma membrane potential $h_{e}$ ) at various concentrations of simulated anaesthesia by assuming that anaesthetics, in addition to increasing the duration of the IPSP, also cause slow adaptive changes in the shape of the mesoscopic firing rate function which relates mean soma membrane potential to mean population firing rate. (2) Actual EEG recorded in children undergoing anaesthesia with the volatile halogenated agent desflurane. (a) 0 MAC (minimum alveolar concentration), (b) $1 \mathrm{MAC}$, (c) $>1 \mathrm{MAC}$ and (d) 2 MAC. Both real and simulated data show that increasing anaesthetic effect is associated with enhanced total EEG power and reductions in mean frequency. Figure adapted from Figs. 6 and 9 of Molaee-Ardekani et al. (2007), with permission

activity (Modica et al. 1990a, b). This pro-convulsant phenomenon is clearly at odds with the suppressive nature of anaesthetic compounds and lacks any clear physiological explanation. Recently, Liley and Bojak (2005) and also Wilson et al. (2006a) provided a dynamical description of this paradoxical phenomenon.

Liley and Bojak (2005) explored the pro-convulsant properties of enflurane by parameterising the IPSP with data from in vivo studies. These findings suggested that a 
distinguishing factor between enflurane and other volatile anaesthetics (e.g. isoflurane) was a significant reduction in IPSP amplitude (Banks and Pearce 1999) even though both agents increase net inhibition. Because of the early developments in IPSP parameterisation Liley and Bojak (2005) were able to systematically explore how independent modulation of the IPSP amplitude influences system dynamics. It was found that anaesthetic agents, such as enflurane, that reduce IPSP amplitude more than other anaesthetics, were more likely to produce EEG activity characteristic of epilepsy (Liley and Bojak 2005). Again Liley and Bojak (2005) used biologically constrained parameter sets which displayed suitable resting EEG spectra. It was found using a bifurcation analysis of a spatially homogeneous model, that isofluranes minimal effect on IPSP amplitude did not produce any progression to system instabilities. However, in over a third of the parameter sets the lower amplitude enflurane IPSPs were shown to induce a strong shift from low firing, low amplitude resting state to an epileptic-like regime (Fig. 8).

At approximately the same time Wilson et al. (2006a) explored the modelling of epileptiform activity induced by anaesthetic agents also. Similar to the work of Liley and Bojak (2005), Wilson et al. (2006a) explored how enflurane IPSP modulation can induce macroscopic excitation. Wilson et al. (2006a) showed too that it was the sensitivity towards IPSP amplitude which made enflurane unique in its effects when compared with isoflurane. An additionally interesting suggestion put forward on the basis of these findings was that the anticonvulsant thiopentone, which has been shown to be proepileptic during administration with low doses of enflurane, may enhance enflurane's influence because of its negligible effect on IPSP amplitude (Wilson et al. 2006a). Finally one more general conclusion made by Wilson et al. (2006a) was the suggestion that the apparent sensitivity of macroscopic dynamics upon the magnitude of the drug induced peak IPSP amplitude may reflect a general phenomenon in which epileptic-like behaviour may be produced by interference with the integrative properties of dendrites.

More generally, mean field models have an immediate potential to assist in the development of physiologically meaningful methods for monitoring anaesthetic depth using EEG. The use of biophysical models of cerebral cortical dynamics in parameterising or otherwise motivating EEG analysis methods for DOA monitoring have the potential to more sensitively detect clinically relevant changes in the EEG given that they are derived from quantitative physiological descriptions of the anaesthetic process. At present most monitoring techniques are based on the construction of discriminating statistical functions from the quantitative analysis of EEG, in large populations of normal and clinical groups undergoing a range of anaesthetic procedures, in order to capture the generalised quantitative changes in the EEG that show systematic variations with anaesthetic action. Despite the early promise of such black-box approaches, recent evidence suggests that they do not provide the clinician with any additional information regarding hypnotic state. In a recent study involving 2,000 patients Avidan et al. (2008) found that the use of bispectral index (BIS) monitoring was not associated with a reduction in the incidence of awareness during anaesthesia when compared to other more standard techniques for monitoring anaesthetic depth. This is significant as the BIS is clinically the (a)

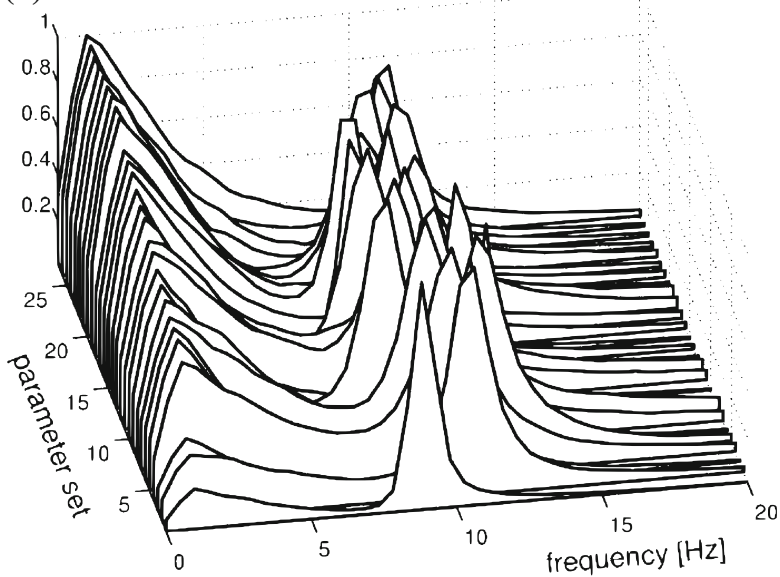

Fig. 8 Liley and Bojak (2005) showed that systematically modulating IPSP shape and amplitude could induce high firing large amplitude activity in a mean field model parameterised to produce electroencephalographically plausible activity ('1/f' low frequency activity and strong alpha resonance). (a) spectra of model EEG activity associated with physiologically plausible parameterisations (b)
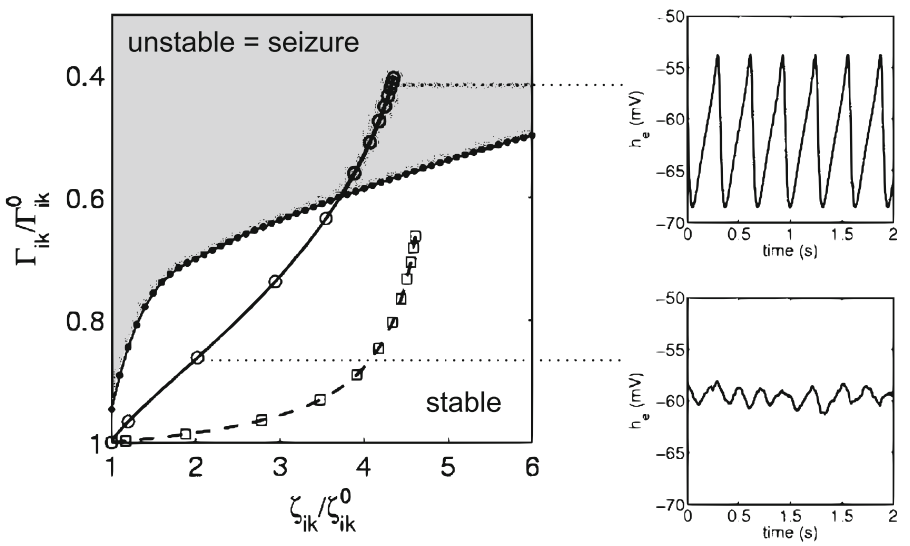

(b) It is predicted that anaesthetics that more rapidly reduce the amplitude of the IPSP $\left(\Gamma_{i k}\right)$ in addition to prolonging it $\left(\zeta_{i k}\right)$, are more likely to produce high firing large amplitude activity electroencephalographic characteristic of epileptiform activity. This may explain why the volatile agent enflurane is paradoxically pro-convulsant. Figure adapted from Figs. 5 and 11 of Liley and Bojak (2005) 
most widely used black-box EEG approach to monitoring anaesthetic depth. Furthermore, the BIS and other heuristic EEG monitoring approaches have been reported to be blind to the effects of a number of anaesthetic agents which are in routine clinical use, including nitrous oxide and the opioids (e.g. Rampil et al. 1998; Anderson and Kakobsson 1992). By using methods of analysis derived from physiological descriptions of the anaesthetic process, quantitative indexing of the EEG for DOA monitoring can perhaps be more robust to the variety of macroscopic physiological consequences of anaesthetic action as observed in the EEG.

\section{Conclusion}

In an attempt to understand the bulk dynamical properties of cortical tissue, researchers have found mean field theories of particular utility due to their ability to incorporate a large range of important physiological phenomena in a relatively tractable mathematical formulation. Through developing biologically plausible models of cortical tissue that span a number of traditionally relevant scales, a quantitative link can be made theoretically between a range of empirical measurements. Theoretically spanning this explanatory gap between molecular/cellular drug action and macroscopic population dynamics assists in dealing with the often inscrutable complexities of brain dynamics. Essential to the success of this approach is the biological plausibility of not only the models but also the parameterisation of drug action. As has been discussed above, some of the first attempts at using such an approach have already been fruitful in the study of anaesthesia. These encouraging results add to the growing utility of dynamical systems theory in the analysis and conceptualisation of neural activity.

However, a number of limitations still remain for neural field approaches. Central to these limitations is the trade off between model utility and model complexity, where maintaining tractability is a serious technical challenge. Firstly, mean field models provide a tractable way of simulating the EEG on large spatial scales, but due to limited physiological and anatomical data often do so by assuming spatially homogeneous parameterisation. Given the well know heterogeneity of neocortical architectonics, improving the spatial plausibility (topology) of these models, and comparing their differences, is a central issue (e.g. Qubbaj and Jirsa 2007). Additionally, the inclusion of more biophysical details to these models often incurs a disproportionate increase in model complexity. Therefore, as part of the improvements to neural field models more generally (biophysical/topological accuracy), attempts should be made to simplify current models where possible in order to limit redundancy and improve parsimony (e.g. Kim and Robinson 2007).
More immediate future developments in mean field or neural mass modelling of cerebral cortex include the extension of the current literature of simulated anaesthesia, especially the exploration of dissociative anaesthetic agents such as ketamine, nitrous oxide and xenon whose pharmacological action is the antagonism of the excitatory glutamatergic NMDA receptor (Hirota 2006). Such developments naturally lead into other domains of brain function, such as learning and memory in the general case of NMDA receptor functioning. Indeed, mean field models of anaesthesia have been extended into sleep (e.g. Wilson et al. 2006b), where the similarities of the EEG spectrum and behavioural state suggest some shared putative mechanisms. Development of these models requires some judicial extension as their already large degrees of freedom must be constrained by empirical data. Some constraints can be provided by utilising mean field models for EEG DOA monitoring as mentioned above, where clinical requirements insist on rapid online assessment of EEG dynamics and therefore limit computational complexity. Together these theoretical and practical improvements may produce a better understanding not only of the quantitative changes in the EEG during anaesthesia but also the physiological processes underlying conscious states.

Acknowledgement This research was supported by Australian Research Council grant number DPO559949.

Open Access This article is distributed under the terms of the Creative Commons Attribution Noncommercial License which permits any noncommercial use, distribution, and reproduction in any medium, provided the original author(s) and source are credited.

\section{References}

Anderson RE, Kakobsson JG (1992) Entropy of EEG during anaesthesia induction: a comparative study with propofol or nitrous oxide as sole agent. Br J Anaesth 92(2):167-170

Aradi I, Erdi P (2006) Computational neuropharmacology: dynamical approaches in drug discovery. Trends Pharmacol Sci 27(5):240 243

Arhem P, Klement G, Nilsson J (2003) Mechanisms of anesthesia: towards integrating network, cellular, and molecular level modeling. Neuropsychopharmacology 28:S40-S47

Avidan M, Zhang L, Burnside B, Finkel K, Searleman A, Selvidge J, Saager L, Turner M, Rao S, Bottros M, Hantler C, Jacobsohn E, Evers A (2008) Anesthesia awareness and the bispectral index. N Engl J Med 358:1097-1108

Banks MI, Pearce RA (1999) Dual actions of volitile anesthetics on GABA(A) IPSCs. Anesthesiology 90:120-134

Basar E (1980) EEG brain-dynamics: relation between EEG and brain evoked potentials. Elsevier, Netherlands

Bojak I, Liley DTJ (2005) Modeling the effects of anaesthesia on the electroencephalogram. Phys Rev E 71:0419021-0419022

Bojak I, Liley DTJ, Cadusch PJ, Cheng K (2004) Electrorhythmogenesis and anaesthesia in a physiological mean field theory. Neurocomputing 58-60:1197-1202 
Breakspear M, Stam CJ (2005) Dynamics of a neural system with a multiscale architecture. Philos Trans R Soc Lond, B, Biol Sci 360:1051-1074

Campagna-Slater V, Weaver DF (2007) Anaesthetic binding sites for etomidate and propofol on a GABAA receptor model. Neurosci Lett 418(1):28-33

Dafilis MP, Liley DTJ, Cadusch PJ (2001) Robust chaos in a model of the electroencephalogram: implications for brain dynamics. Chaos 11(3):474-478

David O, Friston K (2003) A neural mass model for MEG/EEG: coupling and neuronal dynamics. NeuroImage 20(3):1743-1755

Faulkner HJ, Traub RD, Whittington MA (1998) Disruption of synchronous gamma oscillations in the rat hippocampal slice: a common mechanism of anaesthetic drug action. Br J Pharmacol 125(3):483-492

Faulkner HJ, Traub RD, Whittington MA (1999) Anaesthetic/amnesic agents disrupt beta frequency oscillations associated with potentiation of excitatory synaptic potentials in the rat hippocampal slice. Br J Pharmacol 128(8):1813-1825

Feshchenko VA, Veselis RA, Reinsel RA (2004) Propofol-induced alpha rhythm. Neuropsychobiology 50:257-266

Franks NP, Lieb WR (1994) Molecular and cellular mechanisms of general anaesthesia. Nature 367:607-614

Freeman W (1975) Mass action in the nervous system. Academic Press, New York

Freeman WJ (1992) Tutorial on neurobiology: from single neurons to brain chaos. Int J Bifurcat Chaos 2(3):451-482

Freeman WJ (2000) Neurodynamics: an exploration in mesoscopic brain dynamics. Springer-Verlag, New York

Hanna MG (2006) Genetic neurological channelopathies. Nat Clin Pract Neurol 2(5):252-263

Heinke W, Koelsch S (2005) The effects of anesthetics on brain activity and cognitive function. Curr Opin Anaesthesiol 18:625631

Hemmings HC, Akabas MH, Goldstein PA, Trudell BA, Orser JR, Harrison NL (2005) Emerging molecular mechanisms of general anesthetic action. Trends Pharmacol Sci 26(10):503-511

Hirota K (2006) Special cases: ketamine, nitrous oxide and xenon. Best Pract Res Clin Anaesthesiol 20:69-79

Hobson JA, Pace Schott EF (2002) The cognitive neuroscience of sleep:neuronal systems, consciousness and learning. Nat Rev Neurosci 3:679-684

Ishizawa Y (2007) Mechanisms of anesthetic actions and the brain. J Anesth 21(2):187-199

Jensen O, Goel P, Kopell N, Pohja M, Hari R, Ermentrout B (2005) On the human sensorimotor-cortex beta rhythm: sources and modeling. Neuroimage 26:347-355

Jirsa V, Haken H (1996) Field theory of electromagnetic brain activity. Phys Rev Lett 77:960-963

John ER, Prichep LS, Kox W, Valdes-Sosa P, Bosch Bayard J, Aubert E, Tom M, diMichele F, Gugion LD (2001) Invariant reversible qeeg effects of anesthetics. Conscious Cogn 10:165-183

Johnston GAR, Chebib M, Duke RK, Mewett KN, Mitrovic AD, Vandenberg RJ (1999) Medicinal chemistry and molecular pharmacology of GABA receptors and glutamate transporterscomplementary structure-activity relationships. Drug Dev Res $46: 255-260$

Keener J, Sneyd J (2001) Mathematical physiology, 2nd edn. Springer-Verlag, USA

Kelso S (1995) Dynamic patterns: the self organisation of brain and behaviour. MIT Press, USA

Kim JW, Robinson PA (2007) Compact dynamical model of brain activity. Phys Rev E 75:031907

Kishimoto T, Kadoyo C, Sneyd R, Samra SK, Domino EF (1995) Topographic electroencephalogram of propofol-induced conscious sedation. Clin Pharmacol Ther 58(6):666-674
Kiss T, Erdi P (2002) Mesoscopic neurodynamics. Biosystems 64:119-126

Koch C, Segev I (eds) (1998) Methods in neuronal modeling: from ions to networks, 2nd edn. The MIT Press, USA

Kramer M, Szeri A, Sleigh J, Kirsch H (2007) Mechanisms of seizure propagation in a cortical model. J Comput Neurosci 22:63-80

Krasowski MD, Harrison NL (1999) General anaesthetic actions on ligand-gated ion channels. Cell Mol Life Sci 55:1278-1303

Kuizenga K, Kalkman CJ, Hennis PJ (1998) Quantitative electroencephalographic analysis of the biphasic concentration-effects relationships of propofol in surgical patients during extradural analgesia. Br J Anaesth 80:725-732

Kuizenga K, Wierda JMKH, Kalkman CJ (2001) Biphasic EEG changes in relation to loss of consciousness during induction with thiopental, propofol, etomidate, midazolam or sevoflurane. Br J Anaesth 86(3):354-360

Liley DTJ, Bojak I (2005) Understanding the transition to seizure by modeling the epileptiform activity of general anesthetic agents. J Clin Neurophysiol 22(5):300-313

Liley DTJ, Cadusch PJ, Wright JJ (1998) A continuum theory of electro-cortical activity. CalTech, CA

Liley DTJ, Cadusch PJ, Dafilis MP (2002) A spatially continuous mean field theory of electrocortical activity. Network 13:67-113

Liley DTJ, Cadusch PJ, Gray M, Nathan PJ (2003) Drug-induced modification of the system properties associated with spontaneous human electroncephalographic activity. Phys Rev E 68:051096

Lopes da Silva F, Hoeks A, Smits H, Zetterberg LH (1975) Model of brain activity: the alpha rhythm of the thalamus. Biol Cybern 15(1):27-37

Lopes da Silva F, Blanes W, Kalitzin S, Parra J, Suffczynski P, Velis D (2003) Dynamical diseases of brain systems: different routes to epileptic seizures. IEEE Trans Biomed Eng 50:540-548

MacIver MB, Mikulec AA, Amagasu SM, Monroe FA (1996) Volatile anesthetics depress glutamate transmission via presynaptic actions. Anesthesiology 85:823-834

McKernan RM, Whiting PJ (1996) Which GABAA-receptor subtypes really occur in the brain? Trends Neurosci 19:139-143

Modica PA, Tempelhoff R, White PF (1990a) Pro- and anticonvulsant effects of anesthetics (part i). Anesth Analg 70(3):303-315

Modica PA, Tempelhoff R, White PF (1990b) Pro- and anticonvulsant effects of anesthetics (part ii). Anesth Analg 70(4):433-441

Molaee-Ardekani B, Senhadji L, Shamsollahi M, Vosoughi-Vahdat B, Wodey E (2007) Brain activity modeling in general anesthesia: enhancing local mean-field models using a slow adaptive firing rate. Phys Rev E 76:041911

Nelson LE, Guo TZ, Lu J, Saper CB, Franks NP, Maze M (2002) The sedative component of anesthesia is mediated by GABA-A receptors in an endogenous sleep pathway. Nat Neurosci 5(10): 979-984

Nishikawa K, MacIver MB (2000) Excitatory synaptic transmission mediated by NMDA receptors is more sensitive to isoflurane than are non-NMDA receptor-mediated responses. Anesthesiology $92: 228-236$

Nunez P (1981) Electric fields in the brain: the neurophysics of the EEG. Oxford University Press, UK

Nunez PL (1995) Neocortical dynamics and human EEG rhythms. Oxford University Press, USA

Petroff OA (2002) GABA and glutamate in the human brain Neuroscientist 8(6):562-573

Qubbaj MR, Jirsa VK (2007) Neural field dynamics with heterogeneous connection topology. Phys Rev Lett 98(23):238102

Rampil IJ (1998) A primer for EEG signal processing in anesthesia. Anesthesiology 89(4):980-1002

Rampil IJ, Jin Soo K, Lenhardt R, Negishi C, Sessler DI (1998) Bispectral EEG index during nitrous oxide administration. Anesthesiology 89(3):671-677 
Reynolds DS, Rosahl TW, Cirone J, O'Meara GF, Haythornthwaite A, Newman RJ, Myers J, Sur C, Howell O, Rutter AR, Atack J, Macaulay AJ, Hadingham KL, Hutson PH, Belelli D, Lambert JJ, Dawson GR, McKernan R, Whiting PJ, Wafford KA (2003) Sedation and anesthesia mediated by distinct gaba(a) receptor isoforms. J Neurosci 23(24):8608-8617

Robinson PA, Rennie CJ, Wright JJ (1997) Propagation and stability of waves of electrical activity in the cerebral cortex. Phys Rev E 56(1):826-840

Rudolph U, Antkowiak B (2004) Molecular and neuronal substrates for general anaesthetics. Nat Rev Neurosci 5:709-720

Sceniak MP, MacIver MB (2006) Anesthesia in silico. Anesthesiology 104:400-402

Sieghart W, Sperk G (2002) Subunit composition, distribution and function of GABA-A receptor subtypes. Curr Top Med Chem 2:795-816

Stam CJ (2005) Nonlinear dynamical analysis of EEG and MEG: review of an emerging field. Clin Neurophysiol 116:2266-2301

Steyn-Ross ML, Styen-Ross DA, Sleigh JW, Liley DTJ (1999) Theoretical electroencephalogram stationary spectrum for a white-noise-driven cortex: evidence for a general anaestheticinduced phase transition. Phys Rev E 60(6):7299-7311

Steyn-Ross DA, Steyn-Ross ML, Wilcocks LC, Sleigh JW (2001a) Towards a theory of the general-anesthetic-induced phase transition of the cerebral cortex. II. Numerical simulations, spectral entropy, and correlation times. Phys Rev E 64:011918

Steyn-Ross ML, Steyn-Ross DA, Sleigh JW, Wilcocksl LC (2001b) Toward a theory of the general-anesthetic-induced phase transition of the cerebral cortex. I. A thermodynamics analogy. Phys Rev E 64:011971
Steyn-Ross ML, Steyn-Ross DA, Sleigh JW, Whiting DR (2003) Theoretical predictions for spatial covariance of the electroencephalographic signal during the anesthetic-induced phase transition: increased correlation length and emergence of spatial self-organization. Phys Rev E 68:021902

Steyn-Ross ML, Steyn-Ross DA, Sleigh JW (2004) Modelling general anaesthesia as a first-order phase transition in the cortex. Prog Biophys Mol Biol 85(2-3):369-385

Stullken EH, Milde JH, Michenfelder JD, Tinker JH (1977) The nonlinear response of cerebral metabolism to low concentrations of halothane, enflurane, isoflurane, and thiopental. Anesthesiology 46(1):28-34

Traub RD, Jefferys JGR, Whittington MA (1999) Fast oscillations in cortical circuits. The MIT Press, USA

Tsuda N, Hayashi K, Hagihira S, Sawa T (2007) Ketamine, an NMDA-antagonist, increases the oscillatory frequencies of alpha-peaks on the electroencephalographic power spectrum. Acta Anaesthesiol Scand 51(4):472-481

Ujfalussy B, Kiss T, Orban G, Hoffmann WE, Erdi P, Hajos M (2007) Pharmacological and computational analysis of alpha-subunit preferential GABA(A) positive allosteric modulators on the rat septo-hippocampal activity. Neuropharmacology 52(3):733-743

van Gelder TJ (1998) The dynamical hypothesis in cognitive science. Behav Brain Sci 21:1-14

Wilson MT, Sleigh JW, Steyn Ross DA, Steyn Ross ML (2006a) General anesthetic-induced seizures can be explained by a meanfield model of cortical dynamics. Anesthesiology 104(3):588-593

Wilson M, Steyn-Ross D, Sleigh J, Steyn-Ross M, Wilcocks L, Gillies I (2006b) The K-complex and slow oscillation in terms of a mean-field cortical model. J Comput Neurosci 21:243-257 